\title{
Infinitely many shape invariant discrete quantum mechanical systems and new exceptional orthogonal polynomials related to the Wilson and Askey-Wilson polynomials
}

\author{
Satoru Odake ${ }^{*, a}$, Ryu Sasaki ${ }^{\mathrm{b}}$ \\ ${ }^{a}$ Department of Physics, Shinshu University, Matsumoto 390-8621, Japan \\ ${ }^{b}$ Yukawa Institute for Theoretical Physics, Kyoto University, Kyoto 606-8502, Japan
}

\section{'Abstract}

Two sets of infinitely many exceptional orthogonal polynomials related to the Wilson and Askey-Wilson polynomials are presented. They are derived as the eigenfunctions of shape invariant and thus exactly solvable quantum mechanical 'Hamiltonians, which are deformations of those for the Wilson and Askey-Wilson polynomials in terms of a degree $\ell$ $(\ell=1,2, \ldots)$ eigenpolynomial. These polynomials are exceptional in the sense that they start from degree $\ell \geq 1$ and thus not constrained by any generalisation of Bochner's theorem.

Key words: shape invariance, exceptional orthogonal polynomials, discrete quantum mechanics 'PACS: 03.65.-w, 03.65.Ca, 03.65.Fd, 03.65.Ge, 02.30.Ik, 02.30.Gp

\section{Introduction}

Orthogonal polynomials have played important roles $[1,[2]$ on many different stages of mathematics and physics. For example, the matrix models are one of the recent active stages for them in particle physics. As is wellknown there are infinitely many orthogonal polynomials, since they can be constructed by Gram-Schmidt orthonormalisation of the basis $\left\{1, x, x^{2}, \ldots\right\}$ with respect to any given weight (or distribution) $d w(x)$, having finite moments $\int x^{n} d w(x)<\infty, n \in \mathbb{Z}_{+}$. A more practical way of generating orthogonal polynomials $\left\{p_{n}(x)\right\}$ is through the three term recurrence relation (Favard's theorem [3] )

$$
x p_{n}(x)=A_{n} p_{n+1}(x)+B_{n} p_{n}(x)+C_{n} p_{n-1}(x)(n \geq 0),
$$

with $p_{-1}(x)=0$. Here the coefficients $A_{n}, B_{n}$ and $C_{n}$ are real and $A_{n-1} C_{n}>0(n \geq 1)$. Conversely all the orthogonal polynomials starting with degree 0 satisfy the above "three term recurrence relation. Among many orthogonal polynomials, a central role is played by those satisfying (second order) differential or difference equations, in particular those appearing in quantum mechanics or in other dynamical problems. According to Bochner [4], all polynomial solutions of the second order differential equation

$$
\sigma(x) y^{\prime \prime}+\tau(x) y^{\prime}=\lambda_{n} y
$$

are classical orthogonal polynomials, that is the Hermite, Laguerre, Jacobi and Bessel polynomials. Here, $\sigma(x)$ and

\footnotetext{
* Corresponding author.

Email address: odake@azusa.shinshu-u.ac.jp (Satoru Odake)
}

$\tau(x)$ are polynomials in $x$ of $\operatorname{deg}(\sigma) \leq 2, \operatorname{deg}(\tau) \leq 1$. On top of these classical orthogonal polynomials, a variety of orthogonal polynomials, the so-called $(q-)$ Askey scheme of hypergeometric orthogonal polynomials [2, [5, 6, 7], are known to satisfy second order difference equations with pure imaginary or real shifts. They also satisfy restrictions due to various generalisation of Bochner's theorem [8].

In 2008, Gomez-Ullate et al 9] introduced two new orthogonal polynomials satisfying second order differential equations, which are called exceptional $\left(X_{1}\right)$ Laguerre and Jacobi polynomials. They are consistent with Bochner's theorem, since the polynomials start with degree 1 rather than degree 0 constant term. Later reformulation within the framework of quantum mechanics and shape invariance followed in [10, 11]. Recently two sets of infinitely many orthogonal polynomials satisfying second order differential equations are introduced by the present authors [12]. These new polynomials could be called $X_{\ell}$ Laguerre and Jacobi polynomials for each positive integer $\ell$. The $X_{\ell}$ polynomials start with degree $\ell$ and lack the members from degree 0 to degree $\ell-1$ but they form a complete basis. The $\ell=1$ examples are those reported earlier [9, 10, 11].

In this letter we present two new sets of infinitely many orthogonal polynomials satisfying second order difference equations. They could be called exceptional $\left(X_{\ell}\right)$ Wilson and Askey-Wilson polynomials, since they start with degree $\ell \geq 1$. They are complete and satisfy various restrictions due to various generalisation of Bochner's theorem [8]. It is quite natural to expect that these exceptional polynomials (Laguerre, Jacobi, Wilson and Askey-Wilson) will find fruitful applications in many branches of physics and mathematics. The method of deriving these new poly- 
nomials is, as in the cases of the $X_{\ell}$ Laguerre and Jacobi polynomials, shape invariance 13] in quantum mechanics. These polynomials are obtained as the main part of the eigenfunctions of a corresponding Hamiltonian of 'discrete' quantum mechanics (dQM). Thus the orthogonality and completeness of the polynomials are a priori guaranteed. Shape invariance combined with Crum's method [14] or the factorisation method [15] or the supersymmetric quantum mechanics 16 is a sufficient condition for exact solvability of a quantum mechanical Hamiltonian; the entire energy spectrum and the corresponding eigenfunctions can be obtained exactly in an algebraic way. Discrete quantum mechanics (dQM) was introduced in 2004 by the present authors 17, 18 in order to reformulate the theory of hypergeometric orthogonal polynomials [2, 6, 7] within the framework of quantum mechanics, so that various concepts and methods of QM would be available for the theory of these orthogonal polynomials. This revealed the shape invariance of the Hamiltonians for all the hypergeometric orthogonal polynomials [7], ranging from the Meixner-Pollaczek, continuous (dual) Hahn, Wilson, Askey-Wilson and $(q-)$ Racah together with their reductions [19, 20]. Moreover all these dQM systems turn out to be exactly solvable in the Heisenberg picture, too [21]. The positive/negative energy parts of the Heisenberg operator solution define the annihilation/creation operators, which form dynamical symmetry algebras together with the Hamiltonian; for example, the $q$-oscillator algebra 22]. Another benefit of dQM is that it provided many examples of exactly solvable birth and death processes [23]. The Hamiltonians for the two infinite sets of exceptional orthogonal polynomials are obtained by deforming those for the Wilson and Askey-Wilson polynomials in terms of a degree $\ell$ eigenpolynomial with twisted arguments, so that no singularity would appear in the domains where the orthogonality measures are defined. This is essentially the same procedure employed for the exceptional Laguerre and Jacobi polynomials [12]. The necessary technology, the discrete versions of Crum's theorem, was published recently 24.

Here we present our preliminary results on the two sets of infinitely many exceptional orthogonal polynomials and their discrete quantum mechanical formulation, without proof. In the next section, the general setting of dQM and the method of shape invariance are recapitulated. In section three the relationship between the original shape invariance and its multiplicative deformation for the generation of exceptional polynomials is explained for two typical cases of the Wilson and Askey-Wilson polynomials. Sections four and five provide the detailed data of the exceptional Wilson and Askey-Wilson polynomials, respectively. The final section is for a summary and comments.

\section{General setting: shape invariance}

In this letter we consider one dimensional discrete quantum mechanical systems with pure imaginary shifts. The dynamical variables are the real coordinate $x\left(x_{1}<x<\right.$ $x_{2}$ ) and the conjugate momentum $p=-i \partial_{x}$, which are governed by the following generic Hamiltonian:

$$
\begin{gathered}
\mathcal{H} \stackrel{\text { def }}{=} \sqrt{V(x)} e^{\gamma p} \sqrt{V^{*}(x)}+\sqrt{V^{*}(x)} e^{-\gamma p} \sqrt{V(x)} \\
-V(x)-V^{*}(x)=\mathcal{A}^{\dagger} \mathcal{A}, \\
\mathcal{A} \stackrel{\text { def }}{=} i\left(e^{\frac{\gamma}{2} p} \sqrt{V^{*}(x)}-e^{-\frac{\gamma}{2} p} \sqrt{V(x)}\right) \\
\mathcal{A}^{\dagger} \stackrel{\text { def }}{=}-i\left(\sqrt{V(x)} e^{\frac{\gamma}{2} p}-\sqrt{V^{*}(x)} e^{-\frac{\gamma}{2} p}\right) .
\end{gathered}
$$

It is factorised and positive semi-definite. Here the potential function $V(x)$ is an analytic function of $x$ and $\gamma$ is a real constant to be specified for each specific case, see for example (11)-(12) for the $\gamma$ and the domain $x_{1}<x<x_{2}$ for the two cases discussed in this letter. The $*$-operation on an analytic function $f(x)=\sum_{n} a_{n} x^{n}\left(a_{n} \in \mathbb{C}\right)$ is defined as $f^{*}(x)=\sum_{n} a_{n}^{*} x^{n}$, in which $a_{n}^{*}$ is the complex conjugation of $a_{n}$. Since the momentum operator appears in exponentiated forms, the Schrödinger equation $\mathcal{H} \phi_{n}(x)=\mathcal{E}_{n} \phi_{n}(x) \quad(n=0,1,2, \ldots)$, is an analytic difference equation with pure imaginary shifts instead of a differential equation. The groundstate is annihilated by $\mathcal{A}, \mathcal{A} \phi_{0}=0\left(\Rightarrow \mathcal{H} \phi_{0}=0\right)$ and can be chosen 'real', $\phi_{0}^{*}(x)=\phi_{0}(x)$. Throughout this letter we consider those systems which have a square-integrable groundstate together with an infinite number of discrete energy levels: $0=\mathcal{E}_{0}<\mathcal{E}_{1}<\mathcal{E}_{2}<\cdots$.

Shape invariance, a sufficient condition for exact solvability 13], is realised by specific dependence of the potential function on a set of parameters $\boldsymbol{\lambda}=\left(\lambda_{1}, \lambda_{2}, \ldots\right)$, to be denoted by $V(x ; \boldsymbol{\lambda}), \mathcal{A}(\boldsymbol{\lambda}), \mathcal{H}(\boldsymbol{\lambda}), \mathcal{E}_{n}(\boldsymbol{\lambda}), \phi_{n}(x ; \boldsymbol{\lambda})$ etc. The shape invariance condition to be discussed in this letter is [17, 18, 25, 20]

$$
\mathcal{A}(\boldsymbol{\lambda}) \mathcal{A}(\boldsymbol{\lambda})^{\dagger}=\kappa \mathcal{A}(\boldsymbol{\lambda}+\boldsymbol{\delta})^{\dagger} \mathcal{A}(\boldsymbol{\lambda}+\boldsymbol{\delta})+\mathcal{E}_{1}(\boldsymbol{\lambda}),
$$

in which $\boldsymbol{\delta}$ is a certain shift of the parameters and $\kappa$ is a positive constant. This condition is equivalent to the following two relations:

$$
\begin{aligned}
& V\left(x-i \frac{\gamma}{2} ; \boldsymbol{\lambda}\right) V^{*}\left(x-i \frac{\gamma}{2} ; \boldsymbol{\lambda}\right) \\
& \quad=\kappa^{2} V(x ; \boldsymbol{\lambda}+\boldsymbol{\delta}) V^{*}(x-i \gamma ; \boldsymbol{\lambda}+\boldsymbol{\delta}), \\
& \begin{aligned}
V(x & \left.+i \frac{\gamma}{2} ; \boldsymbol{\lambda}\right)+V^{*}\left(x-i \frac{\gamma}{2} ; \boldsymbol{\lambda}\right) \\
& =\kappa\left(V(x ; \boldsymbol{\lambda}+\boldsymbol{\delta})+V^{*}(x ; \boldsymbol{\lambda}+\boldsymbol{\delta})\right)-\mathcal{E}_{1}(\boldsymbol{\lambda}) .
\end{aligned}
\end{aligned}
$$

Then the entire set of discrete eigenvalues and the corresponding eigenfunctions of $\mathcal{H}=\mathcal{H}(\boldsymbol{\lambda})$,

$$
\mathcal{H}(\boldsymbol{\lambda}) \phi_{n}(x ; \boldsymbol{\lambda})=\mathcal{E}_{n}(\boldsymbol{\lambda}) \phi_{n}(x ; \boldsymbol{\lambda}),
$$

is determined algebraically:

$$
\begin{aligned}
& \mathcal{E}_{n}(\boldsymbol{\lambda})=\sum_{s=0}^{n-1} \kappa^{s} \mathcal{E}_{1}(\boldsymbol{\lambda}+s \boldsymbol{\delta}), \\
& \phi_{n}(x ; \boldsymbol{\lambda}) \propto \mathcal{A}(\boldsymbol{\lambda})^{\dagger} \mathcal{A}(\boldsymbol{\lambda}+\boldsymbol{\delta})^{\dagger} \cdots \mathcal{A}(\boldsymbol{\lambda}+(n-1) \boldsymbol{\delta})^{\dagger} \\
& \times \phi_{0}(x ; \boldsymbol{\lambda}+n \boldsymbol{\delta}) .
\end{aligned}
$$




\section{Deformation of shape invariant systems}

In 3.1 we recapitulate two known shape invariant systems, whose eigenfunctions are described by the Wilson polynomial and the Askey-Wilson polynomial. In $\$ 3.2$ we deform them in terms of a degree $\ell$ eigenpolynomial $(\ell=1,2, \ldots)$ and present a new shape invariant system. The general structure is common for these two cases. Explicit expressions of various quantities will be given in 4 and $\$$ respectively.

\section{1. original shape invariant systems}

In 20] various examples of exactly solvable dQM systems are explored with emphasis on shape invariance and closure relation. A closure relation is another sufficient condition for exact solvability, with which the Heisenberg operator solution for the sinusoidal coordinate $\eta(x)$ can be obtained exactly 21 (see 26] for further developments). The examples in [20] are arranged by the sinusoidal coordinates $\left(\eta(x)=x, x^{2}, \cos x\right)$ and the Wilson and Askey-Wilson polynomials are the most generic members for $\eta(x)=x^{2}$ and $\cos x$, respectively,

$$
\begin{aligned}
\text { Wilson : } \eta(x)=x^{2}, & 0<x<\infty, \quad \gamma=1 \\
\text { Askey-Wilson : } \eta(x)=\cos x, & 0<x<\pi, \quad \gamma=\log q .
\end{aligned}
$$

They contain four parameters $\boldsymbol{\lambda}=\left(\lambda_{1}, \lambda_{2}, \lambda_{3}, \lambda_{4}\right)$ (and one more parameter $q(0<q<1)$ in the Askey-Wilson case) and the data for shape invariance are

$$
\boldsymbol{\delta}=\left(\frac{1}{2}, \frac{1}{2}, \frac{1}{2}, \frac{1}{2}\right), \quad \kappa= \begin{cases}1 & : \text { Wilson } \\ q^{-1} & : \text { Askey-Wilson }\end{cases}
$$

It is straightforward to verify that the potential function $V(x ; \boldsymbol{\lambda})$, (57) in 4 and (71) in s5, satisfies the shape invariance conditions (6) - (7).

The groundstate is annihilated by $\mathcal{A}\left(\mathcal{A} \phi_{0}=0\right)$, namely,

$$
\begin{aligned}
& \sqrt{V^{*}\left(x-i \frac{\gamma}{2} ; \boldsymbol{\lambda}\right)} \phi_{0}\left(x-i \frac{\gamma}{2} ; \boldsymbol{\lambda}\right) \\
& =\sqrt{V\left(x+i \frac{\gamma}{2} ; \boldsymbol{\lambda}\right)} \phi_{0}\left(x+i \frac{\gamma}{2} ; \boldsymbol{\lambda}\right),
\end{aligned}
$$

and it satisfies also

$$
\phi_{0}(x ; \boldsymbol{\lambda}+\boldsymbol{\delta})=\varphi(x) \sqrt{V\left(x+i \frac{\gamma}{2} ; \boldsymbol{\lambda}\right)} \phi_{0}\left(x+i \frac{\gamma}{2} ; \boldsymbol{\lambda}\right),
$$

where an auxiliary function $\varphi(x)$ is defined by

$$
\varphi(x) \stackrel{\text { def }}{=} \begin{cases}2 x & : \text { Wilson, } \\ 2 \sin x & : \text { Askey-Wilson. }\end{cases}
$$

The eigenfunctions $\left\{\phi_{n}\right\}$ have the following form:

$$
\phi_{n}(x ; \boldsymbol{\lambda})=\phi_{0}(x ; \boldsymbol{\lambda}) P_{n}(\eta(x) ; \boldsymbol{\lambda}),
$$

where $P_{n}(x ; \boldsymbol{\lambda})$ is a polynomial of degree $n$ in $x$ and is 'real', $P_{n}^{*}(x)=P_{n}(x)$. In the two cases we are considering,
$P_{n}$ is the Wilson polynomial or the Askey-Wilson polynomial, which are orthogonal with respect to the weight function $\phi_{0}(x ; \boldsymbol{\lambda})^{2}$ :

$$
\int_{x_{1}}^{x_{2}} \phi_{0}(x ; \boldsymbol{\lambda})^{2} P_{n}(\eta(x) ; \boldsymbol{\lambda}) P_{m}(\eta(x) ; \boldsymbol{\lambda}) d x=h_{n}(\boldsymbol{\lambda}) \delta_{n m} .
$$

See $\$ 4$ and $\$ 5$ for the explicit forms of the groundstate wavefunction $\phi_{0}(x ; \boldsymbol{\lambda})$, the definition of the polynomial $P_{n}(\eta(x) ; \boldsymbol{\lambda})$ and the normalisation constant $h_{n}(\boldsymbol{\lambda})$.

The action of the operators $\mathcal{A}$ and $\mathcal{A}^{\dagger}$ on the eigenfunction $\phi_{n}$ is

$$
\begin{aligned}
& \mathcal{A}(\boldsymbol{\lambda}) \phi_{n}(x ; \boldsymbol{\lambda})=f_{n}(\boldsymbol{\lambda}) \phi_{n-1}(x ; \boldsymbol{\lambda}+\boldsymbol{\delta}), \\
& \mathcal{A}(\boldsymbol{\lambda})^{\dagger} \phi_{n-1}(x ; \boldsymbol{\lambda}+\boldsymbol{\delta})=b_{n-1}(\boldsymbol{\lambda}) \phi_{n}(x ; \boldsymbol{\lambda}),
\end{aligned}
$$

in which $f_{n}(\boldsymbol{\lambda})$ and $b_{n-1}(\boldsymbol{\lambda})$ are real constants related to $\mathcal{E}_{n}$ as $f_{n}(\boldsymbol{\lambda}) b_{n-1}(\boldsymbol{\lambda})=\mathcal{E}_{n}(\boldsymbol{\lambda})$. Their explicit forms are given in (61) in $\$ 4$ and in (75) in $\$ 5$. The forward shift operator $\mathcal{F}$ and the backward shift operator $\mathcal{B}$ are defined by removing the effects of the groundstate wavefunctions

$$
\begin{aligned}
\mathcal{F}(\boldsymbol{\lambda}) & \stackrel{\text { def }}{=} \phi_{0}(x ; \boldsymbol{\lambda}+\boldsymbol{\delta})^{-1} \circ \mathcal{A}(\boldsymbol{\lambda}) \circ \phi_{0}(x ; \boldsymbol{\lambda}) \\
& =i \varphi(x)^{-1}\left(e^{\frac{\gamma}{2} p}-e^{-\frac{\gamma}{2} p}\right) \\
\mathcal{B}(\boldsymbol{\lambda}) & \stackrel{\text { def }}{=} \phi_{0}(x ; \boldsymbol{\lambda})^{-1} \circ \mathcal{A}(\boldsymbol{\lambda})^{\dagger} \circ \phi_{0}(x ; \boldsymbol{\lambda}+\boldsymbol{\delta}) \\
& =-i\left(V(x ; \boldsymbol{\lambda}) e^{\frac{\gamma}{2} p}-V^{*}(x ; \boldsymbol{\lambda}) e^{-\frac{\gamma}{2} p}\right) \varphi(x),
\end{aligned}
$$

and their action on the polynomial $P_{n}(\eta ; \boldsymbol{\lambda})$ is

$$
\begin{aligned}
& \mathcal{F}(\boldsymbol{\lambda}) P_{n}(\eta(x) ; \boldsymbol{\lambda})=f_{n}(\boldsymbol{\lambda}) P_{n-1}(\eta(x) ; \boldsymbol{\lambda}+\boldsymbol{\delta}), \\
& \mathcal{B}(\boldsymbol{\lambda}) P_{n-1}(\eta(x) ; \boldsymbol{\lambda}+\boldsymbol{\delta})=b_{n-1}(\boldsymbol{\lambda}) P_{n}(\eta(x) ; \boldsymbol{\lambda}) .
\end{aligned}
$$

The difference equation for the polynomials reads

$$
\begin{aligned}
& \widetilde{\mathcal{H}}(\boldsymbol{\lambda}) \stackrel{\text { def }}{=} \mathcal{B}(\boldsymbol{\lambda}) \mathcal{F}(\boldsymbol{\lambda}), \\
& \widetilde{\mathcal{H}}(\boldsymbol{\lambda}) P_{n}(\eta(x) ; \boldsymbol{\lambda})=\mathcal{E}_{n}(\boldsymbol{\lambda}) P_{n}(\eta(x) ; \boldsymbol{\lambda}), \\
& \mathrm{or}: \quad V(x)\left(P_{n}(\eta(x-i \gamma))-P_{n}(\eta(x))\right) \\
& \quad+V^{*}(x)\left(P_{n}(\eta(x+i \gamma))-P_{n}(\eta(x))\right) \\
& \quad=\mathcal{E}_{n} P_{n}(\eta(x)) .
\end{aligned}
$$

In the last equation (27), the explicit parameter $\boldsymbol{\lambda}$ dependence was suppressed for simplicity. The above formulas for the polynomials (21)-(27) are well known [7].

\section{2. new shape invariant systems}

By deforming the shape invariant systems in the previous subsection in terms of a degree $\ell$ eigenpolynomial, we present an infinite number of shape invariant systems indexed by a non-negative integer $\ell=0,1,2, \ldots$ For $\ell=0$, it is the original one. Explicit expressions of various quantities $\left(a_{\ell, n, 1}, a_{\ell, n, 2}, b_{\ell, n, 1}, h_{\ell, n}\right)$ will be given in $\$ 4$ and 马.

In terms of a twist operation $\mathfrak{t}$,

$$
\boldsymbol{\lambda}=\left(\lambda_{1}, \lambda_{2}, \lambda_{3}, \lambda_{4}\right), \quad \mathfrak{t}(\boldsymbol{\lambda}) \stackrel{\text { def }}{=}\left(-\lambda_{1},-\lambda_{2}, \lambda_{3}, \lambda_{4}\right),
$$


we define a degree $\ell$ polynomial $\xi_{\ell}(x)$ from the eigenpolynomial $P_{\ell}(x)$ :

$$
\xi_{\ell}(x ; \boldsymbol{\lambda}) \stackrel{\text { def }}{=} P_{\ell}(x ; \mathfrak{t}(\boldsymbol{\lambda}+(\ell-1) \boldsymbol{\delta})) .
$$

Then we define a potential function $V_{\ell}(x ; \boldsymbol{\lambda})$ and a Hamiltonian $\mathcal{H}_{\ell}(\boldsymbol{\lambda})$ :

$$
\begin{aligned}
& V_{\ell}(x ; \boldsymbol{\lambda}) \stackrel{\text { def }}{=} V(x ; \boldsymbol{\lambda}+\ell \boldsymbol{\delta}) \frac{\xi_{\ell}\left(\eta\left(x+i \frac{\gamma}{2}\right) ; \boldsymbol{\lambda}\right)}{\xi_{\ell}\left(\eta\left(x-i \frac{\gamma}{2}\right) ; \boldsymbol{\lambda}\right)} \\
& \times \frac{\xi_{\ell}(\eta(x-i \gamma) ; \boldsymbol{\lambda}+\boldsymbol{\delta})}{\xi_{\ell}(\eta(x) ; \boldsymbol{\lambda}+\boldsymbol{\delta})}, \\
& V_{\ell}^{*}(x ; \boldsymbol{\lambda})=V^{*}(x ; \boldsymbol{\lambda}+\ell \boldsymbol{\delta}) \frac{\xi_{\ell}\left(\eta\left(x-i \frac{\gamma}{2}\right) ; \boldsymbol{\lambda}\right)}{\xi_{\ell}\left(\eta\left(x+i \frac{\gamma}{2}\right) ; \boldsymbol{\lambda}\right)} \\
& \times \frac{\xi_{\ell}(\eta(x+i \gamma) ; \boldsymbol{\lambda}+\boldsymbol{\delta})}{\xi_{\ell}(\eta(x) ; \boldsymbol{\lambda}+\boldsymbol{\delta})}, \\
& \mathcal{A}_{\ell}(\boldsymbol{\lambda}) \stackrel{\text { def }}{=} i\left(e^{\frac{\gamma}{2} p} \sqrt{V_{\ell}^{*}(x ; \boldsymbol{\lambda})}-e^{\left.-\frac{\gamma}{2} p \sqrt{V_{\ell}(x ; \boldsymbol{\lambda})}\right),}\right. \\
& \mathcal{A}_{\ell}(\boldsymbol{\lambda}) \stackrel{\text { def }}{=}-i\left(\sqrt{V_{\ell}(x ; \boldsymbol{\lambda})} e^{\frac{\gamma}{2} p}-\sqrt{V_{\ell}^{*}(x ; \boldsymbol{\lambda})} e^{-\frac{\gamma}{2} p}\right), \\
& \mathcal{H}_{\ell}(\boldsymbol{\lambda}) \stackrel{\text { def }}{=} \mathcal{A}_{\ell}(\boldsymbol{\lambda})^{\dagger} \mathcal{A}_{\ell}(\boldsymbol{\lambda}) .
\end{aligned}
$$

It is interesting to note that the deformation of the potential function $V(x)$ is multiplicative in contrast to the additive deformation of the prepotential $w(x)$ in the ordinary QM [12]. With an appropriate choice of the range of parameters, the polynomials $\xi_{\ell}(\eta(x) ; \boldsymbol{\lambda})$ and $\xi_{\ell}(\eta(x) ; \boldsymbol{\lambda}+\boldsymbol{\delta})$ have no zero in the rectangular domain in the complex $x$ plane, $x_{1} \leq \operatorname{Re} x \leq x_{2},-\frac{|\gamma|}{2} \leq \operatorname{Im} x \leq \frac{|\gamma|}{2}$ and the Hamiltonian $\mathcal{H}_{\ell}$ is well-defined and hermitian (self-adjoint). The potential function $V_{\ell}(x)$ satisfies the shape invariance conditions (6)-(7),

$$
\begin{aligned}
& V_{\ell}\left(x-i \frac{\gamma}{2} ; \boldsymbol{\lambda}\right) V_{\ell}^{*}\left(x-i \frac{\gamma}{2} ; \boldsymbol{\lambda}\right) \\
& \quad=\kappa^{2} V_{\ell}(x ; \boldsymbol{\lambda}+\boldsymbol{\delta}) V_{\ell}^{*}(x-i \gamma ; \boldsymbol{\lambda}+\boldsymbol{\delta}) \\
& \begin{aligned}
V_{\ell}(x & \left.+i \frac{\gamma}{2} ; \boldsymbol{\lambda}\right)+V_{\ell}^{*}\left(x-i \frac{\gamma}{2} ; \boldsymbol{\lambda}\right) \\
& =\kappa\left(V_{\ell}(x ; \boldsymbol{\lambda}+\boldsymbol{\delta})+V_{\ell}^{*}(x ; \boldsymbol{\lambda}+\boldsymbol{\delta})\right)-\mathcal{E}_{\ell, 1}(\boldsymbol{\lambda}) .
\end{aligned}
\end{aligned}
$$

By using (10) as a Rodrigues type formula, we obtain the complete set of eigenfunctions $(n=0,1,2, \ldots)$ :

$$
\begin{aligned}
& \mathcal{H}_{\ell}(\boldsymbol{\lambda}) \phi_{\ell, n}(x ; \boldsymbol{\lambda})=\mathcal{E}_{\ell, n}(\boldsymbol{\lambda}) \phi_{\ell, n}(x ; \boldsymbol{\lambda}), \\
& \mathcal{E}_{\ell, n}(\boldsymbol{\lambda})=\mathcal{E}_{n}(\boldsymbol{\lambda}+\ell \boldsymbol{\delta}), \\
& \phi_{\ell, 0}(x ; \boldsymbol{\lambda})=\frac{\phi_{0}(x ; \boldsymbol{\lambda}+\ell \boldsymbol{\delta})}{\left|\xi_{\ell}\left(\eta\left(x-i \frac{\gamma}{2}\right) ; \boldsymbol{\lambda}\right)\right|} \xi_{\ell}(\eta(x) ; \boldsymbol{\lambda}+\boldsymbol{\delta}), \\
& \psi_{\ell}(x ; \boldsymbol{\lambda}) \stackrel{\text { def }}{=} \frac{\phi_{0}(x ; \boldsymbol{\lambda}+\ell \boldsymbol{\delta})}{\left|\xi_{\ell}\left(\eta\left(x-i \frac{\gamma}{2}\right) ; \boldsymbol{\lambda}\right)\right|}, \\
& \phi_{\ell, n}(x ; \boldsymbol{\lambda})=\psi_{\ell}(x ; \boldsymbol{\lambda}) P_{\ell, n}(\eta(x) ; \boldsymbol{\lambda}) .
\end{aligned}
$$

Here a degree $\ell+n$ polynomial in $x$, the polynomial eigenfunction $P_{\ell, n}(x ; \boldsymbol{\lambda})$ is given by

$$
\begin{aligned}
P_{\ell, n}(x ; \boldsymbol{\lambda}) \stackrel{\text { def }}{=} & a_{\ell, n}(x ; \boldsymbol{\lambda}) P_{n}(x ; \boldsymbol{\lambda}+\ell \boldsymbol{\delta}) \\
& +b_{\ell, n}(x ; \boldsymbol{\lambda}) P_{n-1}(x ; \boldsymbol{\lambda}+\ell \boldsymbol{\delta}),
\end{aligned}
$$

$$
\begin{aligned}
& a_{\ell, n}(x ; \boldsymbol{\lambda}) \stackrel{\text { def }}{=} \xi_{\ell}(x ; \boldsymbol{\lambda}+\boldsymbol{\delta}) \\
& +a_{\ell, n, 1}(\boldsymbol{\lambda}) \xi_{\ell-1}\left(x ; \boldsymbol{\lambda}+\boldsymbol{\delta}+\boldsymbol{\delta}^{\prime}\right) \\
& \quad+a_{\ell, n, 2}(\boldsymbol{\lambda}) \xi_{\ell-2}\left(x ; \boldsymbol{\lambda}+2 \boldsymbol{\delta}+\boldsymbol{\delta}^{\prime}\right), \\
& b_{\ell, n}(x ; \boldsymbol{\lambda}) \stackrel{\text { def }}{=} b_{\ell, n, 1}(\boldsymbol{\lambda}) \xi_{\ell-1}\left(x ; \boldsymbol{\lambda}+\boldsymbol{\delta}+\boldsymbol{\delta}^{\prime}\right),
\end{aligned}
$$

where $\boldsymbol{\delta}^{\prime}$ is

$$
\boldsymbol{\delta}^{\prime} \stackrel{\text { def }}{=} \mathfrak{t}(\boldsymbol{\delta})=\left(-\frac{1}{2},-\frac{1}{2}, \frac{1}{2}, \frac{1}{2}\right) .
$$

Here the coefficients $a_{\ell, n, 1}(\boldsymbol{\lambda}), a_{\ell, n, 2}(\boldsymbol{\lambda})$ and $b_{\ell, n, 1}(\boldsymbol{\lambda})$ are real. Although the polynomial eigenfunction $P_{\ell, n}(\eta(x) ; \boldsymbol{\lambda})$ is a degree $\ell+n$ polynomial in $\eta(x)$, it has only $n$ zeros in the domain $x_{1}<x<x_{2}$. These polynomials are orthogonal with respect to the weight function $\psi_{\ell}(x ; \boldsymbol{\lambda})^{2}$ :

$\int_{x_{1}}^{x_{2}} \psi_{\ell}(x ; \boldsymbol{\lambda})^{2} P_{\ell, n}(\eta(x) ; \boldsymbol{\lambda}) P_{\ell, m}(\eta(x) ; \boldsymbol{\lambda}) d x=h_{\ell, n}(\boldsymbol{\lambda}) \delta_{n m}$.

They form a complete basis of the Hilbert space just like the Wilson or the Askey-Wilson polynomials $P_{n}(\eta(x) ; \boldsymbol{\lambda})$ in the $\ell=0$ case. It should be stressed that $\psi_{\ell}(x ; \boldsymbol{\lambda})$ is not annihilated by $\mathcal{A}_{\ell}$.

The action of the operators $\mathcal{A}_{\ell}$ and $\mathcal{A}_{\ell}^{\dagger}$ on the eigenfunction $\phi_{\ell, n}$ is

$$
\begin{aligned}
& \mathcal{A}_{\ell}(\boldsymbol{\lambda}) \phi_{\ell, n}(x ; \boldsymbol{\lambda})=f_{\ell, n}(\boldsymbol{\lambda}) \phi_{\ell, n-1}(x ; \boldsymbol{\lambda}+\boldsymbol{\delta}), \\
& \mathcal{A}_{\ell}(\boldsymbol{\lambda})^{\dagger} \phi_{\ell, n-1}(x ; \boldsymbol{\lambda}+\boldsymbol{\delta})=b_{\ell, n-1}(\boldsymbol{\lambda}) \phi_{\ell, n}(x ; \boldsymbol{\lambda}), \\
& f_{\ell, n}(\boldsymbol{\lambda})=f_{n}(\boldsymbol{\lambda}+\ell \boldsymbol{\delta}), \quad b_{\ell, n}(\boldsymbol{\lambda})=b_{n}(\boldsymbol{\lambda}+\ell \boldsymbol{\delta}) .
\end{aligned}
$$

The forward shift operator $\mathcal{F}_{\ell}$ and the backward shift operator $\mathcal{B}_{\ell}$ are defined in a similar way as before

$$
\begin{aligned}
& \mathcal{F}_{\ell}(\boldsymbol{\lambda}) \stackrel{\text { def }}{=} \psi_{\ell}(x ; \boldsymbol{\lambda}+\boldsymbol{\delta})^{-1} \circ \mathcal{A}_{\ell}(\boldsymbol{\lambda}) \circ \psi_{\ell}(x ; \boldsymbol{\lambda}) \\
& =\frac{i}{\varphi(x) \xi_{\ell}(\eta(x) ; \boldsymbol{\lambda})}\left(\xi_{\ell}\left(\eta\left(x+i \frac{\gamma}{2}\right) ; \boldsymbol{\lambda}+\boldsymbol{\delta}\right) e^{\frac{\gamma}{2} p}\right. \\
& \left.\quad-\xi_{\ell}\left(\eta\left(x-i \frac{\gamma}{2}\right) ; \boldsymbol{\lambda}+\boldsymbol{\delta}\right) e^{-\frac{\gamma}{2} p}\right) \\
& \mathcal{B}_{\ell}(\boldsymbol{\lambda}) \stackrel{\text { def }}{=} \psi_{\ell}(x ; \boldsymbol{\lambda})^{-1} \circ \mathcal{A}_{\ell}(\boldsymbol{\lambda})^{\dagger} \circ \psi_{\ell}(x ; \boldsymbol{\lambda}+\boldsymbol{\delta}) \\
& =\frac{-i}{\xi_{\ell}(\eta(x) ; \boldsymbol{\lambda}+\boldsymbol{\delta})}\left(V(x ; \boldsymbol{\lambda}+\ell \boldsymbol{\delta}) \xi_{\ell}\left(\eta\left(x+i \frac{\gamma}{2}\right) ; \boldsymbol{\lambda}\right) e^{\frac{\gamma}{2} p}\right. \\
& \left.\quad-V^{*}(x ; \boldsymbol{\lambda}+\ell \boldsymbol{\delta}) \xi_{\ell}\left(\eta\left(x-i \frac{\gamma}{2}\right) ; \boldsymbol{\lambda}\right) e^{-\frac{\gamma}{2} p}\right) \varphi(x)
\end{aligned}
$$

and their action on the polynomial $P_{\ell, n}(\eta ; \boldsymbol{\lambda})$ is

$$
\begin{aligned}
& \mathcal{F}_{\ell}(\boldsymbol{\lambda}) P_{\ell, n}(\eta(x) ; \boldsymbol{\lambda})=f_{\ell, n}(\boldsymbol{\lambda}) P_{\ell, n-1}(\eta(x) ; \boldsymbol{\lambda}+\boldsymbol{\delta}), \\
& \mathcal{B}_{\ell}(\boldsymbol{\lambda}) P_{\ell, n-1}(\eta(x) ; \boldsymbol{\lambda}+\boldsymbol{\delta})=b_{\ell, n-1}(\boldsymbol{\lambda}) P_{\ell, n}(\eta(x) ; \boldsymbol{\lambda}) .
\end{aligned}
$$

The operator $\widetilde{\mathcal{H}}_{\ell}(\boldsymbol{\lambda})$ acting on the polynomial eigenfunctions is defined by

$$
\begin{array}{rl}
\widetilde{\mathcal{H}}_{\ell}(\boldsymbol{\lambda}) \stackrel{\text { def }}{=} & \mathcal{B}_{\ell}(\boldsymbol{\lambda}) \mathcal{F}_{\ell}(\boldsymbol{\lambda}) \\
=V & V(x ; \boldsymbol{\lambda}+\ell \boldsymbol{\delta}) \frac{\xi_{\ell}\left(\eta\left(x+i \frac{\gamma}{2}\right) ; \boldsymbol{\lambda}\right)}{\xi_{\ell}\left(\eta\left(x-i \frac{\gamma}{2}\right) ; \boldsymbol{\lambda}\right)} \\
& \times\left(e^{\gamma p}-\frac{\xi_{\ell}(\eta(x-i \gamma) ; \boldsymbol{\lambda}+\boldsymbol{\delta})}{\xi_{\ell}(\eta(x) ; \boldsymbol{\lambda}+\boldsymbol{\delta})}\right)
\end{array}
$$




$$
\begin{gathered}
+V^{*}(x ; \boldsymbol{\lambda}+\ell \boldsymbol{\delta}) \frac{\xi_{\ell}\left(\eta\left(x-i \frac{\gamma}{2}\right) ; \boldsymbol{\lambda}\right)}{\xi_{\ell}\left(\eta\left(x+i \frac{\gamma}{2}\right) ; \boldsymbol{\lambda}\right)} \\
\times\left(e^{-\gamma p}-\frac{\xi_{\ell}(\eta(x+i \gamma) ; \boldsymbol{\lambda}+\boldsymbol{\delta})}{\xi_{\ell}(\eta(x) ; \boldsymbol{\lambda}+\boldsymbol{\delta})}\right), \\
\widetilde{\mathcal{H}}_{\ell}(\boldsymbol{\lambda}) P_{\ell, n}(\eta(x) ; \boldsymbol{\lambda})=\mathcal{E}_{\ell, n}(\boldsymbol{\lambda}) P_{\ell, n}(\eta(x) ; \boldsymbol{\lambda}) .
\end{gathered}
$$

\section{Exceptional Wilson polynomials}

We present the data in 93 for the Wilson polynomial.

\section{1. original shape invariant system}

We take the four parameters as follows:

$$
\begin{aligned}
& \boldsymbol{\lambda} \stackrel{\text { def }}{=}\left(a_{1}, a_{2}, a_{3}, a_{4}\right), \quad \operatorname{Re} a_{i}>0 \quad(1 \leq i \leq 4), \\
& \left\{a_{1}^{*}, a_{2}^{*}, a_{3}^{*}, a_{4}^{*}\right\}=\left\{a_{1}, a_{2}, a_{3}, a_{4}\right\} \quad \text { (as a set) }
\end{aligned}
$$

The potential function, ground state, eigenpolynomial, etc. are given by

$$
\begin{aligned}
& V(x ; \boldsymbol{\lambda}) \stackrel{\text { def }}{=} \frac{\prod_{j=1}^{4}\left(a_{j}+i x\right)}{2 i x(2 i x+1)}, \\
& \phi_{0}(x ; \boldsymbol{\lambda}) \stackrel{\text { def }}{=}\left|\frac{\prod_{j=1}^{4} \Gamma\left(a_{j}+i x\right)}{\Gamma(2 i x)}\right| \\
& P_{n}(\eta(x) ; \boldsymbol{\lambda})=W_{n}\left(x^{2} ; a_{1}, a_{2}, a_{3}, a_{4}\right) \stackrel{\text { def }}{=} \prod_{j=2}^{4}\left(a_{1}+a_{j}\right)_{n} \\
& \quad \times{ }_{4} F_{3}\left(\begin{array}{c}
-n, n+b-1, a_{1}+i x, a_{1}-i x \\
a_{1}+a_{2}, a_{1}+a_{3}, a_{1}+a_{4}
\end{array} \mid 1\right), \\
& \mathcal{E}_{n}(\boldsymbol{\lambda})=n(n+b-1), \quad b \stackrel{\text { def }}{=} \sum_{j=1}^{4} a_{j}, \\
& f_{n}(\boldsymbol{\lambda})=-n(n+b-1), \quad b_{n}(\boldsymbol{\lambda})=-1, \\
& h_{n}(\boldsymbol{\lambda})=\frac{2 \pi n !(n+b-1)_{n}}{\Gamma(2 n+b)} \prod_{1 \leq j<k \leq 4} \Gamma\left(n+a_{j}+a_{k}\right) .
\end{aligned}
$$

Here $W_{n}\left(x^{2} ; a_{1}, a_{2}, a_{3}, a_{4}\right)$ is the Wilson polynomial, which is symmetric with respect to $a_{1}, \ldots, a_{4}$.

\section{2. new shape invariant systems}

We generically restrict the original parameter range (56) as follows:

$$
\begin{aligned}
& a_{1}, a_{2} \in \mathbb{R}, \quad\left\{a_{3}^{*}, a_{4}^{*}\right\}=\left\{a_{3}, a_{4}\right\} \quad \text { (as a set), } \\
& 0<a_{j}<\operatorname{Re} a_{k} \quad(j=1,2 ; k=3,4) .
\end{aligned}
$$

The polynomial $\xi_{\ell}(x)$ is

$\xi_{\ell}(x ; \boldsymbol{\lambda})=W_{\ell}\left(x ;-a_{1}-\frac{\ell-1}{2},-a_{2}-\frac{\ell-1}{2}, a_{3}+\frac{\ell-1}{2}, a_{4}+\frac{\ell-1}{2}\right)$,

which is symmetric under $a_{1} \leftrightarrow a_{2}$ and/or $a_{3} \leftrightarrow a_{4}$. It is elementary to show that $\xi_{\ell}(x ; \boldsymbol{\lambda})$ and $\xi_{\ell}(x ; \boldsymbol{\lambda}+\boldsymbol{\delta})$ have no zero on the half real line $0 \leq x<\infty$. The hermiticity of $\mathcal{H}_{\ell}$ requires a stronger condition of no zero in the rectangular domain $-\frac{1}{2} \leq \operatorname{Im} x \leq \frac{1}{2}$. It can be determined purely algebraically for each $\ell$. For the lowest case, $\ell=1$, hermiticity is satisfied by

$$
\left(a_{3}+a_{4}\right)\left(a_{1} a_{2}+\frac{1}{4}\right)<\left(a_{1}+a_{2}\right)\left(a_{3} a_{4}+\frac{1}{4}\right) .
$$

The higher $\ell$ goes the restriction due to hermiticity becomes less stringent.

The real coefficients $a_{\ell, n, 1}, a_{\ell, n, 2}$ and $b_{\ell, n, 1}$ are

$$
\begin{aligned}
& a_{\ell, n, 1}(\boldsymbol{\lambda})=\frac{\ell n\left(a_{1}+a_{2}-a_{3}-a_{4}-\ell+1\right)}{a_{1}+a_{2}-a_{3}-a_{4}-2(\ell-1)} \\
& \quad \times\left(a_{1}+a_{2}+a_{3}+a_{4}+2(n+\ell-1)\right)^{-1} \\
& \quad \times\left(\left(a_{1}+\frac{n}{2}\right)^{2}+\left(a_{2}+\frac{n}{2}\right)^{2}-\left(a_{3}+\frac{n}{2}+\ell-1\right)^{2}\right. \\
& \left.\quad-\left(a_{4}+\frac{n}{2}+\ell-1\right)^{2}\right), \quad(66)
\end{aligned}
$$

The new normalisation constant $h_{\ell, n}(\boldsymbol{\lambda})$ is a simple deformation of the original one $h_{n}(\boldsymbol{\lambda})(\underline{62})$ :

$$
\begin{aligned}
h_{\ell, n}(\boldsymbol{\lambda})= & \frac{\left(a_{1}+a_{2}+n+\ell\right)\left(a_{3}+a_{4}+n+2 \ell-1\right)}{\left(a_{1}+a_{2}+n\right)\left(a_{3}+a_{4}+n+\ell-1\right)} \\
& \times h_{n}(\boldsymbol{\lambda}+\ell \boldsymbol{\delta}) .
\end{aligned}
$$

\section{Exceptional Askey-Wilson polynomials}

We present the data in $₫ 3$ for the Askey-Wilson polynomial.

\section{1. original shape invariant system}

We take the four parameters as follows:

$$
\begin{aligned}
& q^{\boldsymbol{\lambda}} \stackrel{\text { def }}{=}\left(a_{1}, a_{2}, a_{3}, a_{4}\right), \quad\left|a_{i}\right|<1 \quad(i=1, \ldots, 4), \\
& \left\{a_{1}^{*}, a_{2}^{*}, a_{3}^{*}, a_{4}^{*}\right\}=\left\{a_{1}, a_{2}, a_{3}, a_{4}\right\} \quad \text { (as a set), }
\end{aligned}
$$

where $q^{\left(\lambda_{1}, \lambda_{2}, \ldots\right)} \stackrel{\text { def }}{=}\left(q^{\lambda_{1}}, q^{\lambda_{2}}, \ldots\right)$. We have one more parameter $q(0<q<1)$ but its dependence is not explicitly displayed. The potential function, ground state, eigenpolynomial, etc. are given by

$$
\begin{aligned}
& V(x ; \boldsymbol{\lambda}) \stackrel{\text { def }}{=} \frac{\prod_{j=1}^{4}\left(1-a_{j} e^{i x}\right)}{\left(1-e^{2 i x}\right)\left(1-q e^{2 i x}\right)}, \\
& \phi_{0}(x ; \boldsymbol{\lambda}) \stackrel{\text { def }}{=}\left|\frac{\left(e^{2 i x} ; q\right)_{\infty}}{\prod_{j=1}^{4}\left(a_{j} e^{i x} ; q\right)_{\infty}}\right|, \\
& P_{n}(\eta(x) ; \boldsymbol{\lambda})=p_{n}\left(\cos x ; a_{1}, a_{2}, a_{3}, a_{4} \mid q\right) \\
& \quad \stackrel{\text { def }}{=} a_{1}^{-n}\left(a_{1} a_{2}, a_{1} a_{3}, a_{1} a_{4} ; q\right)_{n}
\end{aligned}
$$




$$
\begin{gathered}
\times{ }_{4} \phi_{3}\left(\begin{array}{c}
q^{-n}, a_{1} a_{2} a_{3} a_{4} q^{n-1}, a_{1} e^{i x}, a_{1} e^{-i x} \\
a_{1} a_{2}, a_{1} a_{3}, a_{1} a_{4}
\end{array} \mid q ; q\right), \\
\mathcal{E}_{n}(\boldsymbol{\lambda})=\left(q^{-n}-1\right)\left(1-b q^{n-1}\right), \quad b \stackrel{\text { def }}{=} \prod_{j=1}^{4} a_{j}, \\
f_{n}(\boldsymbol{\lambda})=q^{\frac{n}{2}}\left(q^{-n}-1\right)\left(1-b q^{n-1}\right), \quad b_{n}(\boldsymbol{\lambda})=q^{-\frac{n+1}{2}}, \\
h_{n}(\boldsymbol{\lambda})=2 \pi \frac{\left(b q^{n-1} ; q\right)_{n}\left(b q^{2 n} ; q\right)_{\infty}}{\left(q^{n+1} ; q\right)_{\infty} \prod_{1 \leq j<k \leq 4}\left(a_{j} a_{k} q^{n} ; q\right)_{\infty}} .
\end{gathered}
$$

Here $p_{n}\left(x ; a_{1}, a_{2}, a_{3}, a_{4} \mid q\right)$ is the Askey-Wilson polynomial, which is symmetric with respect to $a_{1}, \ldots, a_{4}$.

\section{2. new shape invariant systems}

We generically restrict the original parameter range (70) as follows:

$$
\begin{aligned}
& a_{1}, a_{2} \in \mathbb{R}, \quad\left\{a_{3}^{*}, a_{4}^{*}\right\}=\left\{a_{3}, a_{4}\right\} \quad \text { (as a set), } \\
& 1>a_{j}>\left|a_{k}\right| \quad(j=1,2 ; k=3,4)
\end{aligned}
$$

The polynomial $\xi_{\ell}(x)$ is

$\xi_{\ell}(x ; \boldsymbol{\lambda})=p_{\ell}\left(x ;\left(a_{1} q^{\frac{\ell-1}{2}}\right)^{-1},\left(a_{2} q^{\frac{\ell-1}{2}}\right)^{-1}, a_{3} q^{\frac{\ell-1}{2}}, a_{4} q^{\frac{\ell-1}{2}} \mid q\right)$,

which is symmetric under $a_{1} \leftrightarrow a_{2}$ and/or $a_{3} \leftrightarrow a_{4}$. It is again elementary to show that $\xi_{\ell}(x ; \boldsymbol{\lambda})$ and $\xi_{\ell}(x ; \boldsymbol{\lambda}+\boldsymbol{\delta})$ have no zero in the real line segment $0 \leq x \leq \pi$. The hermiticity of $\mathcal{H}_{\ell}$ requires a stronger condition of no zero in the rectangular domain $-\frac{|\gamma|}{2} \leq \operatorname{Im} x \leq \frac{|\gamma|}{2}$. It can be determined purely algebraically for each $\ell$. For the lowest case, $\ell=1$, hermiticity is satisfied if

$$
\begin{array}{r}
\left(a_{1}+a_{2}\right)\left(1-a_{3} a_{4}\right)-\left(a_{3}+a_{4}\right)\left(1-a_{1} a_{2}\right) \\
>\left(q^{\frac{1}{2}}+q^{-\frac{1}{2}}\right)\left(a_{1} a_{2}-a_{3} a_{4}\right) .
\end{array}
$$

The higher $\ell$ goes the restriction due to hermiticity becomes the less stringent.

The real coefficients $a_{\ell, n, 1}, a_{\ell, n, 2}$ and $b_{\ell, n, 1}$ are

$$
\begin{aligned}
& a_{\ell, n, 1}(\boldsymbol{\lambda})=\left(q^{n+\ell-1}\left(a_{1}+a_{2}\right)-q^{n+2(\ell-1)}\left(a_{3}+a_{4}\right)\right. \\
& \left.+q^{\ell-1}\left(a_{1}^{-1}+a_{2}^{-1}\right)-\left(a_{3}^{-1}+a_{4}^{-1}\right)\right) \\
& \times \frac{q^{\frac{\ell}{2}-1}\left(1-q^{\ell}\right)\left(1-q^{n}\right)\left(1-a_{1}^{-1} a_{2}^{-1} a_{3} a_{4} q^{\ell-1}\right) a_{3} a_{4}}{\left(1-a_{1}^{-1} a_{2}^{-1} a_{3} a_{4} q^{2(\ell-1)}\right)\left(1-a_{1} a_{2} a_{3} a_{4} q^{2(n+\ell-1)}\right)} \\
& a_{\ell, n, 2}(\boldsymbol{\lambda})=-\prod_{j=1}^{2} \prod_{k=3}^{4}\left(1-a_{j}^{-1} a_{k} q^{\ell-1}\right) \\
& \times \frac{\left(q^{-\ell}-1\right)\left(1-q^{\ell-1}\right)\left(1-q^{n}\right)\left(1-a_{3} a_{4} q^{2(\ell-1)}\right)}{\left(1-a_{1} a_{2} q^{n}\right)\left(1-a_{1}^{-1} a_{2}^{-1} a_{3} a_{4} q^{2(\ell-1)}\right)}, \\
& b_{\ell, n, 1}(\boldsymbol{\lambda})=\left(q^{-\ell}-1\right)\left(1-q^{n}\right) \prod_{j=1}^{2} \prod_{k=3}^{4}\left(1-a_{j} a_{k} q^{n+\ell-1}\right) \\
& \times \frac{\left(1-a_{1} a_{2} q^{n+\ell-1}\right)\left(1-a_{1}^{-1} a_{2}^{-1} a_{3} a_{4} q^{\ell-1}\right)}{\left(1-a_{1} a_{2} q^{n}\right)\left(1-a_{1} a_{2} a_{3} a_{4} q^{2(n+\ell-1)}\right)}
\end{aligned}
$$

The new normalisation constant $h_{\ell, n}(\boldsymbol{\lambda})$ is again a simple deformation of the original one $h_{n}(\boldsymbol{\lambda})$ (76):

$$
\begin{aligned}
h_{\ell, n}(\boldsymbol{\lambda})= & q^{-\ell} \frac{\left(1-a_{1} a_{2} q^{n+\ell}\right)\left(1-a_{3} a_{4} q^{n+2 \ell-1}\right)}{\left(1-a_{1} a_{2} q^{n}\right)\left(1-a_{3} a_{4} q^{n+\ell-1}\right)} \\
& \times h_{n}(\boldsymbol{\lambda}+\ell \boldsymbol{\delta}) .
\end{aligned}
$$

\section{3. limit to Wilson case}

It is well known that the Wilson polynomials are obtained from the Askey-Wilson polynomials in a certain limit 7] together with the corresponding potential functions, $\mathcal{A}$ and $\mathcal{A}^{\dagger}$ operators and the Hamiltonians [20]. This is the reason why various formulas for the exceptional Askey-Wilson polynomials and those of the exceptional Wilson polynomials are common as shown in 33.2 , In fact we show that the same limiting procedure will connect various quantities between the two exceptional polynomials. Following the Askey-Wilson $\rightarrow$ Wilson limiting arguments in 20], let us introduce the rescaled variable $x^{\mathrm{W}}=\frac{L}{\pi} x$ and set $\gamma=-\frac{\pi}{L}, q=e^{-\frac{\pi}{L}}$. This implies $0<x<\pi \Leftrightarrow 0<x^{\mathrm{W}}<L, p^{\mathrm{W}}=\frac{\pi}{L} p, e^{\gamma p}=e^{-p^{\mathrm{W}}}$. We write the $x$-dependence explicitly as $\mathcal{H}_{\ell}=\mathcal{H}_{\ell}(x ; \boldsymbol{\lambda})$, $\mathcal{A}_{\ell}=\mathcal{A}_{\ell}(x ; \boldsymbol{\lambda})$, etc. By setting $\boldsymbol{\lambda}=\left(a_{1}^{\mathrm{W}}, a_{2}^{\mathrm{W}}, a_{3}^{\mathrm{W}}, a_{4}^{\mathrm{W}}\right)$ $\left(b=\sum_{j=1}^{4} a_{j}^{\mathrm{W}}\right)$, the desired limit is achieved by $L \rightarrow \infty$ $(q \rightarrow 1)$ :

$$
\begin{aligned}
& \lim _{L \rightarrow \infty}(1-q)^{-3 \ell} \xi_{\ell}(\eta(x) ; \boldsymbol{\lambda})=\xi_{\ell}^{\mathrm{W}}\left(\eta^{\mathrm{W}}\left(x^{\mathrm{W}}\right) ; \boldsymbol{\lambda}\right), \\
& \lim _{L \rightarrow \infty}(1-q)^{-3(\ell+n)} P_{\ell, n}(\eta(x) ; \boldsymbol{\lambda})=P_{\ell, n}^{\mathrm{W}}\left(\eta^{\mathrm{W}}\left(x^{\mathrm{W}}\right) ; \boldsymbol{\lambda}\right), \\
& \lim _{L \rightarrow \infty}(q ; q)_{\infty}^{3}(1-q)^{3-b+\ell} \psi_{\ell}(x ; \boldsymbol{\lambda})=\psi_{\ell}^{\mathrm{W}}\left(x^{\mathrm{W}} ; \boldsymbol{\lambda}\right), \\
& \lim _{L \rightarrow \infty}(1-q)^{-2} V_{\ell}(x ; \boldsymbol{\lambda})=V_{\ell}^{\mathrm{W} *}\left(x^{\mathrm{W}} ; \boldsymbol{\lambda}\right), \\
& \lim _{L \rightarrow \infty}(1-q)^{-1} \varphi(x)=\varphi^{\mathrm{W}}\left(x^{\mathrm{W}}\right), \\
& \lim _{L \rightarrow \infty}(1-q)^{-1} \mathcal{A}_{\ell}(x ; \boldsymbol{\lambda})=-\mathcal{A}_{\ell}^{\mathrm{W}}\left(x^{\mathrm{W}} ; \boldsymbol{\lambda}\right), \\
& \lim _{L \rightarrow \infty}(1-q)^{-2} \mathcal{H}_{\ell}(x ; \boldsymbol{\lambda})=\mathcal{H}_{\ell}^{\mathrm{W}}\left(x^{\mathrm{W}} ; \boldsymbol{\lambda}\right), \\
& \lim _{L \rightarrow \infty}(1-q) \mathcal{F}_{\ell}(x ; \boldsymbol{\lambda})=-\mathcal{F}_{\ell}^{\mathrm{W}}\left(x^{\mathrm{W}} ; \boldsymbol{\lambda}\right), \\
& \lim _{L \rightarrow \infty}(1-q)^{-3} \mathcal{B}_{\ell}(x ; \boldsymbol{\lambda})=-\mathcal{B}_{\ell}^{\mathrm{W}}\left(x^{\mathrm{W}} ; \boldsymbol{\lambda}\right), \quad \text { etc. }
\end{aligned}
$$

where the superscript ${ }^{\mathrm{W}}$ denotes the corresponding quantity in $\$ 4.2$. The orthogonality (46) also correctly reduces to that of 4.2 .

By construction dQM reduces to the ordinary QM in the limit when the shifts become infinitesimal. Thus the Jacobi polynomials are obtained from the Askey-Wilson polynomials and the Laguerre polynomials from the Wilson polynomials in certain limits [7, 25]. It is possible to write down the limit formulas connecting various quantities of the $X_{\ell}$ Askey-Wilson polynomials to those of the $X_{\ell}$ Jacobi polynomials, as well as those connecting the $X_{\ell}$ Wilson and Laguerre polynomials. We will defer these rather technical results to a later publication. 


\section{Summary and Comments}

By deforming two well-known shape invariant discrete quantum mechanical systems, corresponding to the Wilson and Askey-Wilson polynomials, in terms of their eigenpolynomials, two infinite sets of shape invariant systems are obtained. Their eigenpolynomials form new types of orthogonal polynomials, called $X_{\ell}$ Wilson and AskeyWilson polynomials, starting with degree $\ell(\ell=1,2, \ldots)$, which is the degree of the polynomial used for the deformation. By construction these exceptional orthogonal polynomials do not satisfy the three term recurrence relation (1) but they form complete sets due to the hermiticity (self-adjointness) of the Hamiltonian. The allowed parameter ranges are determined by the purely algebraic conditions that the polynomials used for deformation should not have a zero in the rectangular domain $x_{1} \leq \operatorname{Re} x \leq x_{2}$, $-\frac{|\gamma|}{2} \leq \operatorname{Im} x \leq \frac{|\gamma|}{2}$. The explicit ranges for the lowest case $\ell=1$ are shown in (65) and (79). The higher $\ell$ goes the restriction due to hermiticity becomes the less stringent. It is also possible to find the allowed parameter ranges outside of those generically specified ranges (63) and (77). It would be interesting to try to perform similar deformation of other shape invariant systems; for example, the Meixner-Pollaczek, continuous (dual) Hahn and various reductions of the Askey-Wilson polynomials. More interesting would be to formulate the exceptional (q-)Racah polynomials, the most generic examples of discrete quantum mechanics with real shifts. It is a good challenge to clarify various properties of these new polynomials, e.g. generating functions, the Gram-Schmidt construction, substitutes of the three term recurrence relations (10), etc., and to pursue possible physical applications.

We thank A. Khare for useful discussion. This work is supported in part by Grants-in-Aid for Scientific Research from the Ministry of Education, Culture, Sports, Science and Technology, No.19540179.

\section{References}

[1] G.Szegö, Orthogonal polynomials, 4th edition, Amer. Math. Soc. Colloq. Pub. 23 Providence, R.I. (1975).

[2] G. E. Andrews, R. Askey and R. Roy, Special Functions, Encyclopedia of mathematics and its applications, Cambridge, (1999).

[3] T.S. Chihara, An Introduction to orthogonal polynomials, Gordon and Breach, New York (1978).

[4] S. Bochner, Math. Zeit. 29 (1929) 730.

[5] A.F. Nikiforov, S. K. Suslov, and V.B. Uvarov, Classical Orthogonal Polynomials of a Discrete Variable, Springer, Berlin, (1991).

[6] M. E. H. Ismail Classical and quantum orthogonal polynomials in one variable, Encyclopedia of mathematics and its applications, Cambridge, (2005).

[7] R. Koekoek and R.F. Swarttouw, arXiv:math.CA/9602214

[8] R. Askey and J.Wilson, Mem. Amer. Math. Soc. 54 No.319 (1985) 1; F. Alberto Grünbaum and L. Haine, J. Comput. Appl. Math. 68 (1996) 103; M. E. H. Ismail, J. Comput. Appl. Math. 159 (2003) 319324; L. Vinet and A. Zhedanov, J. Comput. Appl. Math. 211 (2008) 45, arXiv:0712.0069[math.CA].
[9] D. Gómez-Ullate, N. Kamran and R. Milson, arXiv:0805.3376 [math-ph]; J. Math. Anal. Appl. 359 (2009) 352, arXiv:0807. 3939 [math-ph]; J. Phys. A37 (2004) 10065.

[10] C. Quesne, J. Phys. A41 (2008) 392001, arXiv:0807.4087 3 [quant-ph]; SIGMA 5 (2009) 084, arXiv:0906.2331 [math-ph] .

[11] B. Bagchi, C. Quesne and R. Roychoudhury, Pramana J. Phys. 73 (2009) 337-347, arXiv:0812.1488[quant-ph].

[12] S. Odake and R.Sasaki, Phys. Lett. B679 (2009) 414, arXiv: 0906.0142 [math-ph].

[13] L. E. Gendenshtein, JETP Lett. 38 (1983) 356.

[14] M. M. Crum, Quart. J. Math. Oxford Ser. (2) 6 (1955) 121, arXiv:physics/9908019

[15] L. Infeld and T. E. Hull, Rev. Mod. Phys. 23 (1951) 21.

[16] See, for example, a review: F. Cooper, A. Khare and U. Sukhatme, Phys. Rep. 251 (1995) 267.

[17] S. Odake and R. Sasaki, J. Nonlinear Math. Phys. 12 Suppl. 1 (2005) 507, arXiv:hep-th/0410102

[18] S. Odake and R.Sasaki, J. Math. Phys. 46 (2005) 063513, arXiv: hep-th/0410109

[19] S. Odake and R.Sasaki, J. Math. Phys. 49 (2008) 053503, arXiv:0712.4106 [math. CA].

[20] S. Odake and R. Sasaki, Prog. Theor. Phys. 119 (2008) 663, arXiv: 0802.1075 [quant-ph].

[21] S. Odake and R.Sasaki, J. Math. Phys. 47 (2006) 102102, arXiv: quant-ph/0605215; Phys. Lett. B641 (2006) 112, arXiv: quant-ph/0605221.

[22] S. Odake and R. Sasaki, Phys. Lett. B663 (2008) 141, arXiv: 0710.2209 [hep-th].

[23] R. Sasaki, J. Math. Phys. 50 (2009) 103509, arXiv:0903.3097 [math-ph].

[24] P. Gaillard and V. B. Matveev, J. Phys. A42 (2009) 404009; S. Odake and R. Sasaki, Prog. Theor. Phys. 122 (2009) 1067, arXiv:0902.2593 [math-ph].

[25] S. Odake and R. Sasaki, Prog. Theor. Phys. 114 (2005) 1245, arXiv: hep-th/0512155

[26] S. Odake and R. Sasaki, arXiv:0903.2604 [math-ph]. 